\title{
Modeling the fluid-dynamics and oxygen consumption in a porous scaffold stimulated by cyclic squeeze pressure
}

\author{
Marco Ferroni ${ }^{a}$, Serena Giusti ${ }^{b}$, Diana Nascimento $^{c}$, Ana Silva $^{c}$, Federica Boschetti ${ }^{a}$, \\ Arti Ahluwalia ${ }^{b}, *$ \\ a Laboratory of Biological Structure Mechanics, Chemistry, Materials and Chemical Engineering Department "Giulio Natta”, Politecnico di Milano, Piazza \\ Leonardo da Vinci 32, 20133 Milan, Italy \\ ${ }^{\mathrm{b}}$ Research Center E. Piaggio, University of Pisa, Via Diotisalvi 2, 56122 Pisa, Italy \\ ' INEB-Instituto Nacional de Engenharia Biomédica, Universidade do Porto, Portugal E' ICBAS- Instituto de Ciências Biomédicas Abel Salazar, $4150-180$ Porto, \\ Portugal
}

\begin{abstract}
The architecture and dynamic physical environment of tissues can be recreated in-vitro by combining 3D porous scaffolds and bioreactors able to apply controlled mechanical stimuli on cells. In such systems, the entity of the stimuli and the distribution of nutrients within the engineered construct depend on the micro-structure of the scaffolds. In this work, we present a new approach for optimizing computational fluid-dynamics (CFD) models for the investigation of fluid-induced forces generated by cyclic squeeze pressure within a porous construct, coupled with oxygen consumption of cardiomyocytes. A 2D axial symmetric macro-scaled model of a squeeze pressure bioreactor chamber was used as starting point for generating time dependent pressure profiles. Subsequently the fluid movement generated by the pressure fields was coupled with a complete 3D micro-scaled model of a porous protein cryogel. Oxygen transport and consumption inside the scaffold was evaluated considering a homogeneous distribution of cardiomy- ocytes throughout the structure, as confirmed by preliminary cell culture experiments. The results show that a 3D description of the system, coupling a porous geometry and time dependent pressure driven flow with fluid-structure-interaction provides an accurate and meaningful description of the microenvironment in terms of shear stress and oxygen distribution than simple stationary 2D models.
\end{abstract}

\section{Abbreviations:}

$\mathrm{CDF}$, computational fluid-dynamics;

microCT, micro-computed tomography;

FSI, fluid-structure interactions;

$S^{2} \mathrm{PR}$, sensorized squeeze pressure bioreactor;

SQPR, squeeze pressure bioreactor;

GTA, glutaraldehyde;

ALE, arbitrary Lagrangian-Eulerian;

OC, oxygen consumption;

OC-S, oxygen consumption in static conditions;

OC-D, oxygen consumption in dynamic conditions;

LF, laminar flow;

BDF, backward differentiation formula.

\section{Introduction}

Current tissue engineering strategies are aimed at in-vitro recapitulation of the mechanical and the biochemical cues of the physiological environment in terms of both tissue architecture and physical stimuli [1]. In this context, porous 3D constructs are considered as excellent substitutes for cell culture, as they are able to provide structural, mechanical and architectural templates for cell attachment and tissue formation while maintaining acceptable mass transport [2-4]. Moreover, the combined use of 3D structures in bioreactors is fundamental for recreating the physiological milieu, improving the exchange of nutrients and metabolites as well as providing dynamic molecular and physical regulatory signals $[5,6]$. This is particularly important for load-bearing or actuating tissues like bone or cardiac tissue, which require specific stimuli in order to maintain their differentiated state in-vitro.

Focusing on cardiac tissue engineering, several studies have demonstrated the role of mechanical forces and fluid motion on the organization and function of cardiomyocytes as well as on cardiovascular development [7-9]. In particular, fluid motion increases oxygen and nutrient transport [10], promoting homogenous cell growth in the scaffold as well as the maintenance of cell function and contractility [11,12], whereas mechanical forces can activate mechanotransduction pathways and induce cell alignment and cytoskeletal re-organization [13]. In a previous study, we reported that a cyclic hydrodynamic squeeze pressure can be used for inducing phenotypic differentiation in cardiac cells seeded on 2D collagen sandwiches [14]. However, 3D scaffolds as far more appropriate for regenerative applications as 2D constructs do not adequately replicate the structure and mechanical properties of the

native tissue [6].
E-mail address: arti.ahluwalia@centropiaggio.unipi.it, arti.ahluwalia@ing.unipi.it (A. Ahluwalia). 
When bioreactors are used in combination with porous scaffolds, the entity and the distribution of fluid-induced forces inside the structure are very complex, as they not only depend on the working conditions of the system but also on 3D microstructural parameters of the scaffold, like pore diameter, porosity, and pore interconnectivity [15]. Computational methods are powerful tools able to estimate local fluid-induced shear, pressure and velocity fields in bioreactor environments. Previous studies have shown that the fluid-dynamic environment within a porous structure can be represented using time-invariant computational fluiddynamics (CFD) models, in order to quantify the fluid-structure in- teractions and the mechanical stimuli applied to cells. These CFD models follow two different approaches: i) 3D solid models of the real scaffold microstructure, reconstructed from microcomputed tomography (microCT) images [15-17]; ii) a simplified and idealized geometry obtained from the macro-characteristics of the scaffold (pore size, interconnected porosity) [18-20]. Moreover, fluid movement also plays a fundamental role in the transport of nutrients within the construct, which is a critical factor for modulating cell growth and functions. Several mathematical and com- putational models have been implemented to combine the diffusion of nutrients, like oxygen or glucose, with cell consump- tion rates, both in static [21-23] and dynamic conditions [24-27].

However, very few studies have attempted to combine fluid perfusion through the interconnected pores and cell consumption of oxygen considering the effective micro-architecture of the scaffold [15,16], instead of its global characteristics (i.e. porosity, permeability) [25-27]. This is mainly due to the extremely high computational costs required for such analyses, particularly if further combined with fluid-structure interactions (FSI) during the mechanical stimulation.

The aim of this study was thus to implement a series of computationally optimized 2D axial symmetric macro-scaled and 3D micro-scaled models of porous cryogels in the presence of a complex fluid-dynamic microenvironment. In particular the investigation was focused on the evaluation of the physical and biochemical stimuli imposed by cyclic pressure waves and pulsatile me- dia flow generated in a sensorized squeeze pressure bioreactor ( ${ }^{2} \mathrm{PR}$ ) [28]. Our preliminary cell culture experiments in the bioreactor confirmed that cardiac cells in gelatin cryogel scaffolds sub- ject to a $24 \mathrm{~h}$ long stimulation remain highly viable and are able to proliferate.

In order to predict the levels of shear stress and hydrodynamic pressure acting on cells within the construct and quantify oxygen transport and consumption inside the scaffold, the hydrodynamic stimuli generated by the bioreactor was modeled using a new computational approach. The stepwise approach can be used to investigate and understand the effects of complex stimuli imposed by dynamic cell culture devices on 3D tissue constructs.

\section{Materials and methods}

\subsection{The $S^{2} P R$ bioreactor}

The $S^{2} P R$ is a sensorized version of the SQPR (squeeze pressure bioreactor), a stimulation chamber designed to impose a cyclic, hydrodynamic and contactless overpressure on cell cultures using a simple vertical piston movement [28]. The entity of the stimulus depends principally on the piston velocity and the vertical space in the meatus between the two approaching surfaces. The SQPR is able to generate local overpressures between 0.5 and $8 \mathrm{kPa}$, as well as a pulsatile flow of cell culture media through the scaffold. As a result, cells seeded in the $3 \mathrm{D}$ constructs are subjected to mechanical forces, which enhance the diffusion of oxygen and nutrients through the structure.

The architecture of the bioreactor has been described in our previous publication [28]. In this work, it has been modified to incorporate a force and a position sensor, assuring high precision and control of the piston movement with an accuracy of $5 \mu \mathrm{m}$. (Fig. 1). The force sensor (Flexiforce A201, Tekscan, Inc. MA, USA) is placed under the sample brace so as to detect any contact between the piston and the scaffold (Fig. 1.A). To improve the sensitivity of the Flexiforce, the base of the $S^{2} P R$ is designed to maximize the resolution and the sensitivity of the sensor: the bottom of the base is provided with a $5 \mathrm{~mm}$ diameter puck to optimize the contact surface with the sensing area of the sensor. This configuration significantly increases its sensitivity $(230 \pm 20$ bits/N with the puck; $128 \pm 6.2$ bits/N without the puck).

In addition, the stepper motor (L4118S144, Nanotec Electronic $\mathrm{GmbH} \&$ Co, Munich, Germany) driving the piston is provided with
A

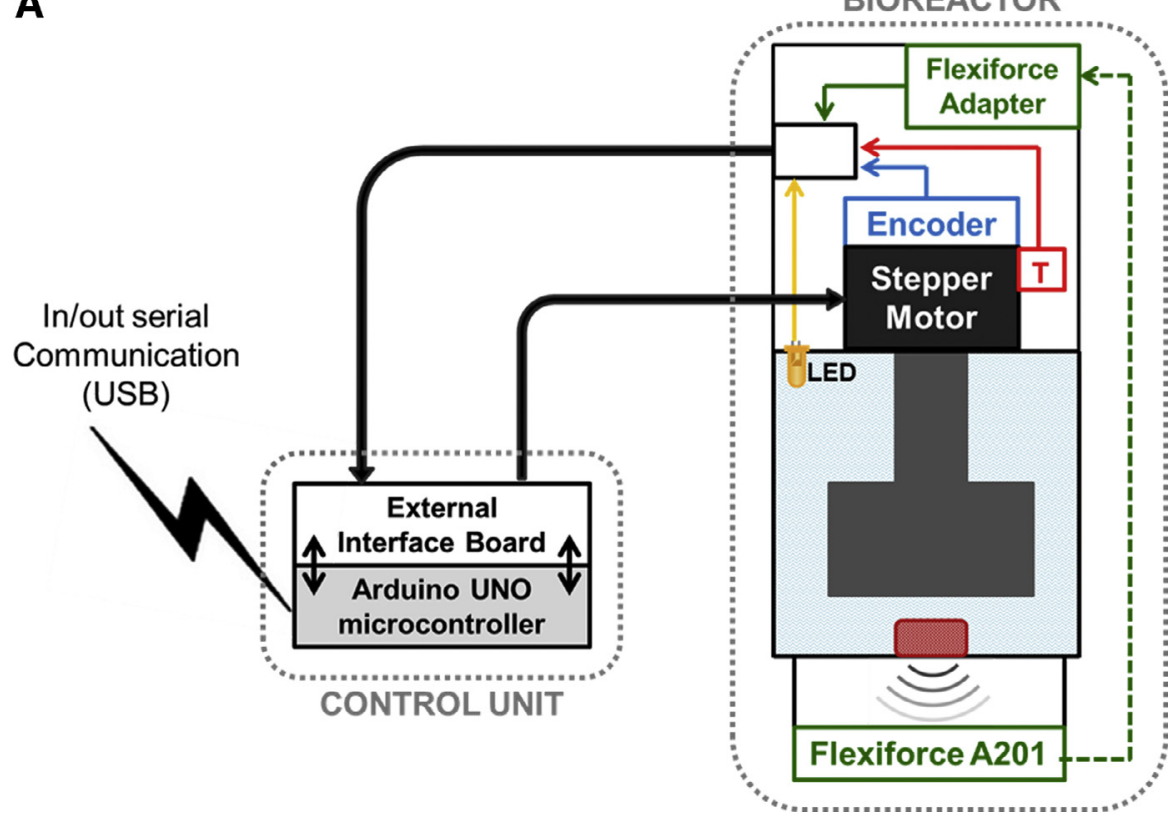

B

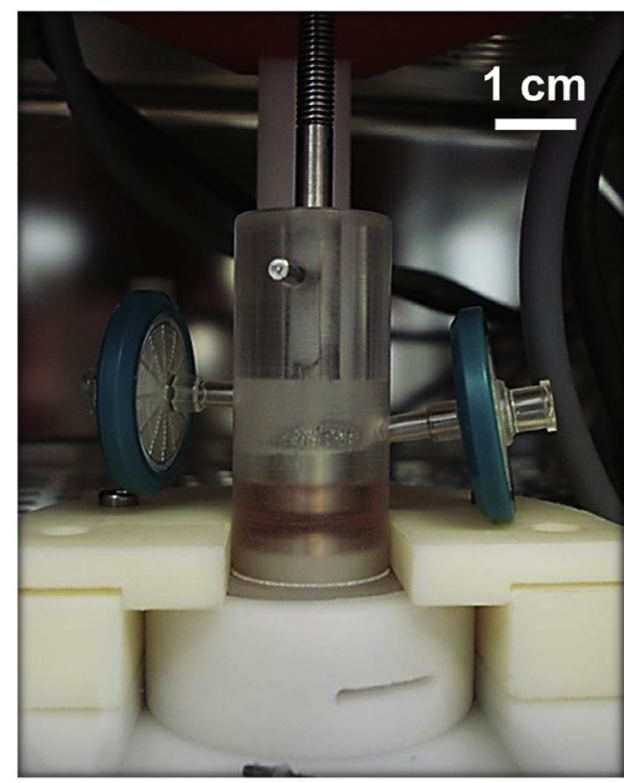

Fig. 1. (A) Functional block diagram showing the architecture of the $S^{2} P R$ bioreactor. (B) A detail of the $S^{2} P R$ bioreactor chamber and support base. 
an incremental encoder embedded in the motor shaft to monitor the position and the movement of the piston in real time. Signals from the force and position sensors are continuously matched using a microcontroller (Arduino UNO, Italy), in order to automatically set the starting position of the piston at the beginning of the experiment and control its movement during the stimulation. The presence of the force sensor and the encoder enable real-time evaluation of construct stiffness through compressive tests.

The $S^{2} \mathrm{PR}$ is provided with a dedicated graphical user-interface from which the user can set all the stimulation parameters such as the maximum value of the hydrodynamic pressure applied, the frequency of the stimulus and the duration of the experiment.

\subsection{Gelatin cryogel}

All reagents were all purchased from Sigma-Aldrich (Milan, Italy), unless otherwise specified. Porous disc-shaped $(10 \mathrm{~mm}$ diameter, $1 \mathrm{~mm}$ height) gelatin cryogels were fabricated from gelatin $5 \% \mathrm{w} / \mathrm{v}$ (Type A gelatin from pig skin) in deionized water, freezedried and chemically crosslinked with $1 \mathrm{mM}$ glutaraldehyde (GTA), as previously reported $[29,30]$. The scaffolds were characterized in an aqueous environment using compressive testing, water imbibition and Darcy's law flow to obtain quantitative values of elastic modulus, porosity and permeability respectively. The porosity and elastic modulus were evaluated as described in Spinelli et al. [30], giving an elastic modulus in the linear region of small deformations (up to $2 \%$ ) of $3.8 \pm 0.37 \mathrm{kPa}$ and a porosity of $91.01 \pm 0.57 \%$. The permeability $(K)$ of the scaffolds was determined by measuring the flow rate $(Q)$ through $n=5$ scaffolds keeping a constant pressure difference $(\Delta P)$ of about 550 Pa across the sample and applying Darcy's law:

$K=\mu_{F} \frac{L}{S} \frac{Q}{(\Delta P)}$

where $\mu_{\mathrm{F}}$ is the viscosity of water and $\mathrm{L}$ and $\mathrm{S}$ are the height and the cross-section of sample. The measured permeability was $(13.01 \pm 3.99) \times 10^{-12} \mathrm{~m}^{2}$. MicroCT images of the material were used to estimate the structural properties of the construct: they are random pore and isotropic, with an average fiber thickness of $45 \pm 17 \mu \mathrm{m}$ and an average pore diameter of $139 \pm 39 \mu \mathrm{m}$ (data not shown).

\subsection{Cell culture}

HL-1 myocytes were cultured following the protocols and media cocktail described in Claycomb et al. [31]. Before cell seeding the cryogel scaffolds were re-hydrated for $24 \mathrm{~h}$ at $37^{\circ} \mathrm{C}$ in media, and pre-coated with fibronectin/gelatin overnight at $37^{\circ} \mathrm{C}$, as previously reported for HL-1 cells in 3D structures [32,33]. Then $40 \mu \mathrm{L}$ of cell suspension at a concentration of $1.5 \times 10^{7}$ cells $/ \mathrm{mL}$ was pipetted onto each construct, obtaining a concentration of $4 \times 10^{6}$ cells $/ \mathrm{cm}^{3}$. Scaffolds were incubated for $30 \mathrm{~min}$ to allow preliminary cell attachment, then $500 \mu \mathrm{L}$ of complete medium was added to each well to completely submerge the constructs. They were cultured for 4 days at $37^{\circ} \mathrm{C}$ and $5 \% \mathrm{CO}_{2}$, before placing in the bioreactors.

Four experiments were run in parallel in four $S^{2}$ PR chambers. A scaffold was placed in each sterile bioreactor chamber filled with $2.5 \mathrm{~mL}$ of complete media. The constructs were subject to $24 \mathrm{~h}$ of squeeze pressure with a frequency of $1 \mathrm{~Hz}$ cycling meatus heights from 1200 to 200 microns with an approaching and receding velocity of 11.6 and $2 \mathrm{~mm} / \mathrm{s}$ respectively. Fig. 3.C shows the displacement of the piston during the approach, recede and hold phases of a cycle. Static controls were performed in a 12-well plate with the same amount of media. At the end of the stimulation, cell spreading and viability in the 3D structures was assessed with the alamar blue assay and calcein staining, and imaged using a confocal

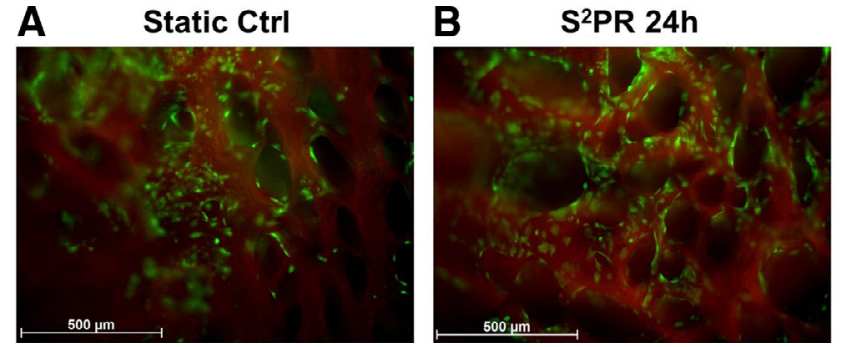

Fig. 2. Calcein staining on the gelatin cryogel. (A) In static conditions and (B) after the $24 \mathrm{~h}$ long stimulation.

microscope (Nikon A1 Confocal Microscope System). As shown in Fig. 2, HL-1 cells are able to withstand the hydrodynamic stimulation and maintain high viability. Moreover, confocal imaging showed that the cells had migrated and spread into the scaffold, showing a better organization and distribution in the dynamic experiments. To characterize the nature of the dynamic stimulus and its effect on the cell microenvironment, a computational approach was used to quantify the squeeze pressure inside the porous structure and the oxygen gradients in the construct.

\subsection{Computational models}

Computational models were implemented using Comsol Multiphysics 4.3b (COMSOL Inc., Burlington, MA, USA) running on a Windows PC with 16 GB RAM. In order to optimize and refine the computational studies step-by-step, simple time-dependent 2D CFD models were first realized to evaluate pressure and velocity profiles around the construct due to movement of the piston. Here, fluid-dynamics in the bioreactor chamber and inside the scaffold was modeled using Navier-Stokes equations in the free-media and Brinkman equations for the porous media. Then, the fluid-dynamic results from the $2 \mathrm{D}$ models were applied to an idealized 3D geometry of the porous scaffold [18] to evaluate the oxygen consumption and transport within the construct and the shear-stress inside the porous structure during the stimulation considering the fluid-structure interaction. In fact, because of the compliant nature of the scaffold, the characteristics of the fluid domain alone cannot properly describe the fluid-dynamics within the scaffold unless combined with a FSI approach. A complete description of mathematical formulations used in all the models is provided in the Supplementary Information (SI1).

The same conditions as in the cell culture experiments were imposed in all models: cell culture media essentially consists of water and dissolved salts, small molecules and serum, thus it can be considered as an incompressible, homogeneous and Newtonian fluid with a density of $1029 \mathrm{~kg} / \mathrm{m}^{3}$ and a dynamic viscosity of $1.2 \times 10^{-3} \mathrm{~Pa}$. For the FSI model we considered the scaffold as homogeneous, isotropic and elastic within the regime of small deformations as observed experimentally [30].

Simulations were computed using the time-dependent solver based on the BDF (backward differentiation formula) method, with maximum order set to two and free steps taken by the solver. Convergence criteria were based on relative and absolute tolerances, so the time-dependent solver goes to the next step when the estimated local error vector in the integration step is less than or equal to the sum of the absolute and relative tolerance $\times$ the vector of the dependent variables.

\subsubsection{Fluid-dynamic $2 \mathrm{D}$ models}

Assuming axial symmetry, the geometry of the 2D models was reduced to a transverse section of the bioreactor chamber (Fig. 3.A). In order to reproduce the fluid-dynamic conditions of the 
Table 1

Boundary conditions set in the 2D models. See Fig. 3.A for identification of side numbers.

\begin{tabular}{llll}
\hline & Side number & Type of boundary condition & Boundary condition \\
\hline \multirow{4}{*}{ CFD } & 2,11 & Symmetry & Axial symmetry \\
& $3,5,13$ & Inlet/Outlet & $p=p_{2}, v=v_{2}$ \\
& 6 & Wall & No slip \\
& $7,8,9,10$ & Outlet & Pressure $p=0$ \\
& Wall & Moving wall $v(\mathrm{t})$ \\
\hline \multirow{3}{*}{ ALE } & $2,3,4,5,6,11,13$ & Prescribed mesh displacement & $d_{\mathrm{r}}=d_{\mathrm{z}}=0$ \\
& $7,8,9,10$ & Prescribed mesh displacement & $d_{\mathrm{r}}=0 d_{\mathrm{z}}=v(\mathrm{t})$ \\
& 1 & Prescribed mesh displacement & Free \\
\hline
\end{tabular}

A

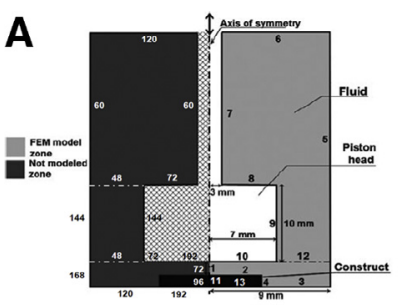

B

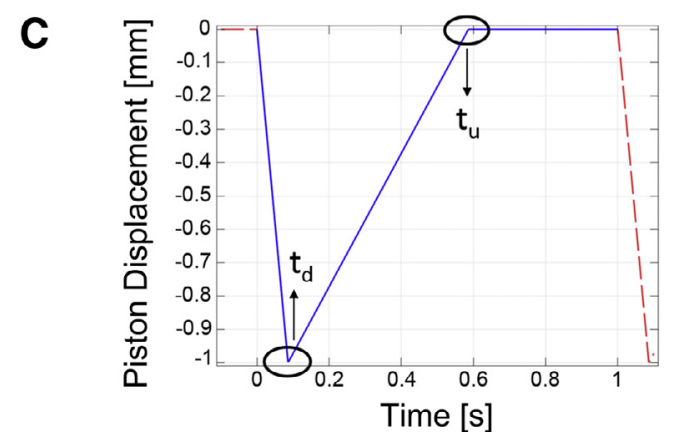

Fig. 3. (A) Modeled region of the $S^{2} P R$ bioreactor (light gray) with corresponding number of boundary conditions (on the right) and number of edge elements on boundaries (on the left). (B) Schematic representation of the scaffold in the $S^{2} P R$ chamber (not scaled), pointing out the directions $p_{R}$ and $p_{A}$ where the pressure profiles are averaged and exported from 2D to 3D models. (C) Piston movement during the squeeze pressure cycle.

squeeze pressure stimulus, three different physics modes were implemented: (i) Navier-Stokes equations for an incompressible fluid were used for the laminar fluid flow in the bioreactor chamber, (ii) Brinkman equations were applied for flow in the porous scaffold, (iii) the Arbitrary Lagrangian-Eulerian (ALE) method was used to model piston movement. The scaffold domain was defined using the measured porosity and permeability values for gelatin cryogels [30]. To couple the 3 modes, the velocity field evaluated in the laminar flow regime was applied as a boundary condition of the Brinkman module. The moving mesh was used to consider changes in the geometry of the meatus due to the cyclic piston motion which generates the squeeze stimulus.

As previously described, the piston moves vertically in the fluid-filled chamber, approaching and receding from the base without touching the scaffold (Fig. 3A and 3C). To apply the velocity profile as a moving wall boundary condition, sharp discontinuities at times $t_{\mathrm{d}}$ and $t_{\mathrm{u}}$, corresponding to the lower and uppermost piston excursion limits respectively, were first smoothed using second order polynomial functions.

Boundary conditions imposed in the 2D model are summarized in Table 1. A mapped mesh of 66,528 elements was obtained by fixing the number of edge elements on boundaries, as reported in Fig. 3A. The 2D models were run using the PARDISO solver.

\subsubsection{D models with idealized porous structure}

The time-dependent 3D models were built using an idealized geometry of the fluid domain, starting from the measured pore architecture of the constructs. A simplified geometry based on the radius and height of the whole structure and the dimension of interconnected pores in axial $\left(R_{\mathrm{CZ}}\right)$, circumferential $\left(R_{\mathrm{cC}}\right)$ and radial directions $\left(R_{\mathrm{Cr}}\right)$ was generated as summarized in Table 2. Previous investigations have shown that this method can be applied to porous structures as an alternative to microCT reconstruction, in order to simplify computational analysis [16,18,34,35].

Two different 3D models were implemented in order to evaluate the oxygen distribution inside a construct seeded with cardiomyocytes and the shear stress imposed on the cells during the $\mathrm{S}^{2} \mathrm{PR}$ stimulation, respectively. The domains of all the 3D models were discretized based on a free tetrahedral meshing method. The software allows the definition of common mesh parameters (e.g. maximum and minimum mesh element size) as well as the resolution of narrow regions and curvature factor. These latter parameters enforce a refinement in case of narrow regions and high curvature values, respectively.

In the first oxygen consumption (OC) analysis we only considered a $10^{\circ}$ sector of the fluid domain in the cylindrical scaffold (Fig. 4.A). Oxygen diffusion and convection as well as consumption were considered, comparing both the static (OC-S) and dynamic (OC-D) conditions during the squeeze pressure stimulation. In the OC-D approach we applied pressure profiles, exported from the 2D model (Fig. 4.B-C), as inlet boundary conditions respectively at the top and the sides of the construct. Specifically, the pressure profiles exported from the 2D model are space and time dependent. To simplify the computation, the pressure values were taken as the space averaged profiles along the radial direction $p_{R}(t)$ and the axial direction $p_{A}(t)$, as outlined in the supplementary information and illustrated in Fig. 3.B. In both the OC models, a constant oxygen concentration of $0.21 \mathrm{~mol} / \mathrm{m}^{3}$ was imposed in the scaffold domain at $t=0$ and as a boundary condition at the top and sides of the scaffold. Oxygen consumption was implemented as a mass outflux $R\left(\mathrm{c}\right.$ ) (in moles $/ \mathrm{m}^{2} / \mathrm{s}$ from the total internal surface area, $A_{\text {int }}$ (Table 2), of the scaffold, using the Michaelis Menten kinetics according to the following expression:

$R(c)=\rho_{\text {sup }} \frac{\left(V_{\max } \times c\right)}{K_{m}+c}$

where $V_{\max }$ is in $\mathrm{mol} /($ cells $\cdot \mathrm{s}), K_{\mathrm{m}}$ and $c$ are in $\mathrm{mol} / \mathrm{m}^{3}$ and the cell surface density $\rho_{\text {sup }}$ was set at 30,000 cells $/ \mathrm{cm}^{2}$, a typical value for cardiomyocytes reported in the literature [36].

The mesh of the OC-S and OC-D models were built defining parameters such as maximum and minimum element size, maximum element growth rate, curvature factor and resolution of narrow regions. These values allowed a mesh with just over $10^{6}$ tetrahedral elements to be obtained without any problem of convergence, reducing the computational costs. The model was solved using the MUMPS solver. 
Table 2

List of parameters used in the 2D and 3D models for defining scaffold geometry, fluid properties and oxygen transport and consumption.

\begin{tabular}{lll}
\hline Parameter & Value & Description \\
\hline$R$ & $5 \times 10^{-3} \mathrm{~m}$ & Porous scaffold radius \\
$H$ & $1 \times 10^{-3} \mathrm{~m}$ & Porous scaffold height \\
$R_{\mathrm{cz}}$ & $75 \times 10^{-6} \mathrm{~m}$ & Channel radius in axial direction \\
$R_{\mathrm{cc}}, R_{\mathrm{cr}}$ & $58 \times 10^{-6} \mathrm{~m}$ & Channel and torus radius in radial and circumferential directions \\
$A_{\mathrm{int}}$ & $0.0132 \mathrm{~m}^{2}$ & Internal surface area of the scaffold in OC models \\
$P$ & $1029 \mathrm{~kg} / \mathrm{m}^{3}$ & Culture medium density [28] \\
$M$ & $1.2 \times 10^{-3} \mathrm{~Pa} \cdot \mathrm{s}$ & Culture medium dynamic viscosity \\
$\rho_{\text {sup }}$ & $30,000 \mathrm{cells} / \mathrm{cm}^{2}$ & Surface density of cardiomyocytes [36] \\
$V_{\max }$ & $5.44 \times 10^{-17} \mathrm{~mol} /($ cells $\cdot \mathrm{s})$ & Higher reaction velocity [38] \\
$K_{\mathrm{m}}$ & $3.79 \times 10^{-3} \mathrm{~mol} / \mathrm{m}^{3}$ & Concentration at half saturation [38] \\
$D$ & $2.7 \times 10^{-9} \mathrm{~m}^{2} / \mathrm{s}$ & Oxygen diffusion coefficient in culture medium [38] \\
\hline
\end{tabular}

The aim of the second 3D study was to simulate the interaction between the media and the porous cryogel during cyclic stimulation in the bioreactor using the FSI approach in order to evaluate the shear stress applied to the cell culture. Here, the reference geometry is slightly different from that used in the oxygen consumption analysis. Due to the high complexity in implementing a computational fluid-structure interaction model, we considered a $1 \mathrm{~mm}$ side cube at the center of the scaffold instead of the triangular slice with the same structure used for the transport models. Given the symmetry of the system we focused on a quarter of the cube corresponding to a parallelepiped. Symmetry conditions were imposed at the two internal surfaces of the parallelepiped, while a single pressure profile $p_{\mathrm{R}}(\mathrm{t})$ was applied to the top and the two lateral faces (Fig. 4.D). To determine the importance of the fluid-structure interaction on the evaluation of the effective shear-stress inside the porous structure, the FSI model was compared to a simple laminar flow (LF) model with the same scaffold geometry and fluid properties. The mesh consisted in 217,173 free tetrahedral elements, and the model was solved using the PARDISO solver.

\section{Results}

\subsection{D computational modeling}

The results of the $2 \mathrm{D}$ models permit the evaluation of pressure and flow velocity at specific time-points. Specifically, the changes in fluid motion were analyzed at discrete time points in terms of pressure and velocity during the generation of the squeeze pressure by the $S^{2} P R$ piston, focusing on the instant when the piston is at its lowest position (time $t_{\mathrm{d}}$ ) and highest position (time $t_{\mathrm{u}}$ ) (see Fig. 3.C).

Fig. 5.A shows the distribution of the pressure on the upper face of the scaffold at $t_{\mathrm{d}}$, while the velocity fields at $t_{\mathrm{d}}$ and $t_{\mathrm{u}}$ are reported in Fig. $5 \mathrm{~B}$ and $5 \mathrm{C}$. Pressure profiles $p_{\mathrm{R}}(\mathrm{t})$ and $p_{\mathrm{A}}(\mathrm{t})$ (Fig. 4. B-C) show a typical peak due to the quick piston movement toward the scaffold (approaching phase), as reported in our previous work [28]. The first smaller peak at $t=0$ in Fig. 4.B was also observed experimentally and is probably due to fluid inertia when the piston starts its approach. A more complete description of the pressure and velocity profiles is given in the supplementary information.
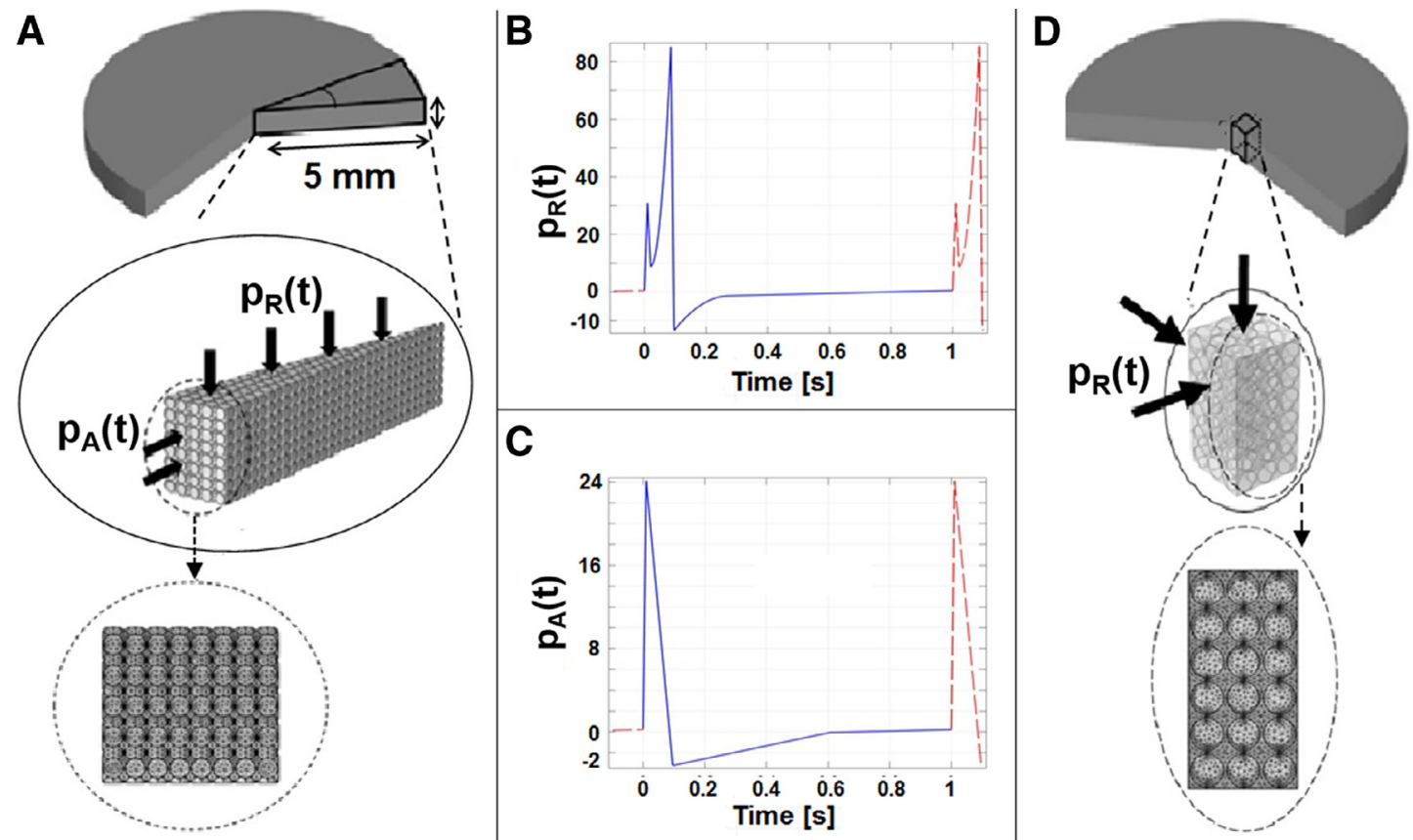

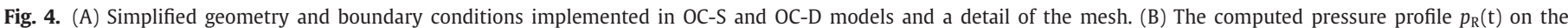

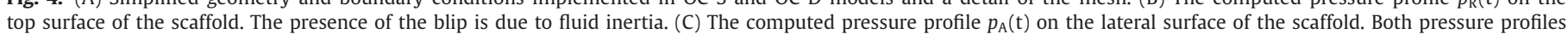
were obtained as solutions of the 2D model. (D) Simplified geometry and boundary conditions implemented in FSI and LF models and a detail of the mesh. 
A

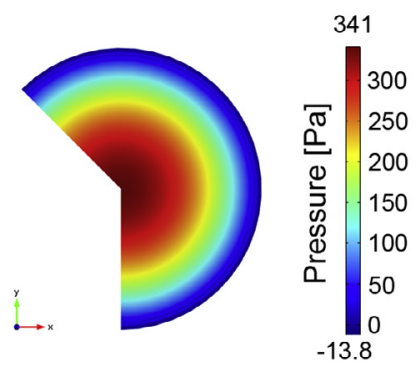

B

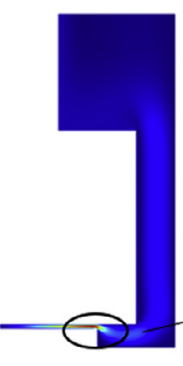

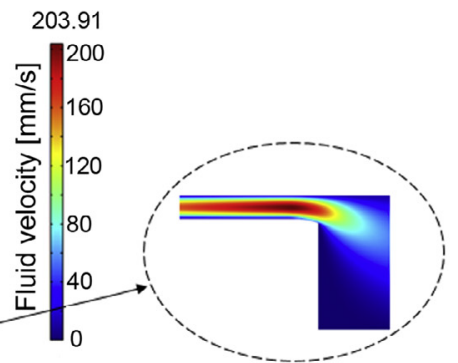

C

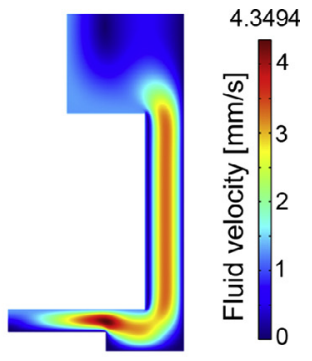

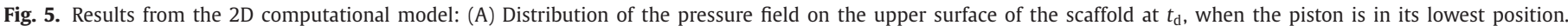

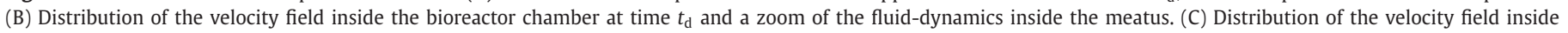
the bioreactor chamber at $t_{\mathrm{u}}$, when the piston is in its highest position.

A

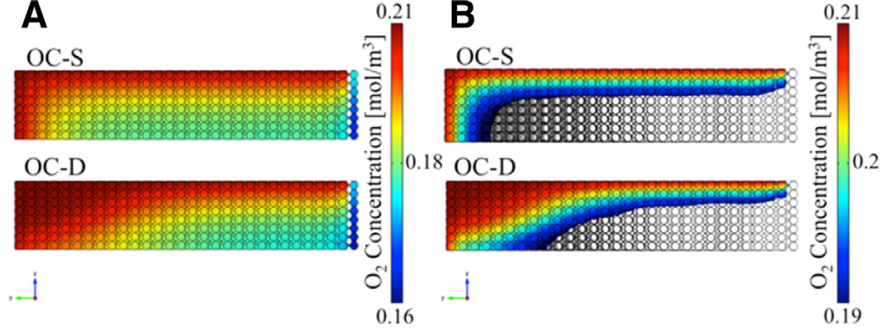

Fig. 6. (A) Oxygen concentration in the scaffold after $200 \mathrm{~s}$ in the OC-S (on the top) and the OC-D (on the bottom) model. (B) Comparison between OC-S (on the top) and OC-D (on the bottom) showing the extent of scaffold where the oxygen concentrations are between 0.19 and $0.21 \mathrm{~mol} / \mathrm{m}^{3}$.

\subsection{D computational modeling}

Space averaged pressure profiles $p_{R}(\mathrm{t})$ and $p_{\mathrm{A}}(\mathrm{t})$ from the 2D models were used as inlet boundary conditions for the OC-D model to evaluate the effect of the hydrodynamic stimulus applied by the bioreactor on transport and oxygen consumption. A transport model in static conditions without the pressure-driven convective contribution (OC-S) was developed in parallel to determine how the dynamic stimulus changes the oxygen content within the porous scaffold.

Fig. 6 reports the oxygen concentration after $200 \mathrm{~s}$ of simulation both in the absence and in the presence of the hydrodynamic stimulus. The choice of this time of simulation is a good compromise between computational costs and reliable solutions, as the difference between the oxygen concentration values after 200 and 3600 s respectively is less than $10 \%$. In Fig. 6.B the same graph as in Fig. 6.A is plotted with a cut-off of $0.19 \mathrm{~mol} / \mathrm{m}^{3}$ oxygen $(90 \%$ of the maximum value $0.21 \mathrm{~mol} / \mathrm{m}^{3}$ ) to better highlight the difference between the dynamic and static conditions.

The scaffold strain computed by the FSI model is maximum in the axial direction, but never greater than $1 \%$, indicating that the non-contact squeeze pressure causes negligible pore deformation and the scaffold remains within the linear elastic region [30]. The FSI model also confirms the suitability of the cyclic stimulation induced by the $S^{2} P R$ bioreactor in terms of shear stress evaluated at the solid-fluid interface. Fig. 7A and 7B show the shear stress (viscosity $\times$ velocity gradient) and velocity maps inside the scaffold calculated by the FSI model. In Fig. 7.B, velocity arrows indicate the direction of fluid motion in the FSI at $t_{\mathrm{d}}$, showing that the fluid penetrates inside the scaffold from the upper surface of the porous scaffold, and comes out from all the pores at the sides. Average values are significantly $\left(10^{3}\right.$-fold) lower than the values computed by the LF model. The Reynolds number in the scaffold (calculated based on the hydraulic diameter and characteristic length
A

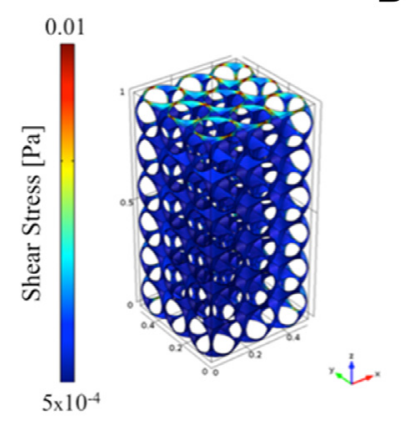

Fig. 7. (A) Shear stress values exported from FSI models. (B) Arrows representing the effective fluid motion inside the scaffold at time $t_{\mathrm{d}}$.

of the pores) was around $2 \times 10^{-5}$ throughout its volume and 0.3 on the surfaces exposed to moving fluid (top and sides).

\section{Discussion}

Our initial cell culture experiments established that cardiomyocytes in the bioreactor are able to colonize porous scaffolds and maintain their viability, indicating that the dynamic microenvironment is suitable for the regeneration of cardiac tissue. To quantify the stimuli applied to cardiomyocytes seeded on the scaffold we modeled the hydrodynamic environment in the $S^{2} \mathrm{PR}$ bioreactor using computational methods with a stepwise approach. Starting with 2D axial symmetric models, we coupled Navier-Stokes equations for fluid-dynamics with the Brinkman equation to consider macro-properties of the scaffold and the ALE method for implementing mesh movement and changes in the geometry of the meatus during squeeze pressure stimulation. Then, 3D models were implemented with a simplified and idealized scaffold geometry. Finally, the oxygen concentration distribution and shear stresses inside the construct were computed by using the pressure profiles from the $2 \mathrm{D}$ model as boundary conditions.

The 2D models highlight the importance of implementing the real time-dependent movement of the piston inside the bioreactor chamber in the presence of a permeable and porous scaffold, with both a qualitative and quantitative improvement of the results with respect to the stationary model reported in De Maria et al. [28]. The non-stationary analysis takes into account the inertial forces of the fluid during cyclic stimulation, and shows that the greatest variations in pressure take place when the piston changes direction (at times $t_{\mathrm{d}}$ and $t_{\mathrm{u}}$ in Fig. 3.C). As we can see in Fig. 5, the culture media goes out from the meatus when the piston moves down $\left(t_{\mathrm{d}}\right.$, Fig. 5.B), whereas it slowly flows back into the meatus from 
the lateral sides of the chamber when the piston recedes from the scaffold ( $t_{\mathrm{u}}$, Fig. 5.C).

Our approach implements 2D models with space and time dependent fluid-dynamics and then extends to more complex 3D time-dependent models with both fluid-dynamics and cell oxygen consumption. In fact, although cell metabolism and growth are critically dependent on oxygen concentration, only a few studies deal with computational models of oxygen transport and consumption [21-27].

The 3D simulations considered a well-defined scaffold with specific values of porosity, permeability and pore size in all three dimensions so as to develop a simplified geometry of a porous cryogel $[15,18,30]$. A model in static conditions (OC-S) was developed to verify that the hydrodynamic stimuli used as inlet in the OC-D model permits better nutrient transport after $24 \mathrm{~h}$ in the $S^{2} \mathrm{PR}$ bioreactor, particularly in the peripheral zones of the construct (Fig. 6). This suggests that the stimulus is not strong enough to drive the fluid inside the whole 3D structure. These results were confirmed by the FSI model: as expected, the highest shear stress values are in the upper zone of the scaffold when the piston is at its lowest and the medium is forced to enter the pores (Fig. 7.A). The values of shear stress are below those considered damaging to cardiac cells (about $0.1 \mathrm{~Pa}$ ) [37]. Our 3D models also demonstrated the need to implement a FSI model when a fluid-induced stimulus is applied to a complex porous scaffold. In fact, the simple laminarflow conditions applied to both the 2D and 3D models gave 1000 fold higher average shear stress values (up to 1 and $6 \mathrm{~Pa}$ in 2D and LF models respectively)-in the range of those known to compromise cell vitality. The high vitality and cardiomyocyte densities observed in the cell culture experiments suggest that the implementation of a 3D fluid-structure interaction model is mandatory for accurately mimicking the fluid-dynamics inside the bioreactor.

A further advancement of this work is the implementation of a model scaffold with a simplified geometry: this feature allows to us to change its micro-structural properties and consequently evaluate the capacity of the bioreactor to perfuse a porous structure. Using this approach we can optimize the scaffold design for different applications. Moreover, compared to previous reports which only deal with uncoupled fluid-structure models [17], our fluidstructure interaction model considers the mechanical forces acting on both the solid and fluid domain of the 3D matrix.

In conclusion, the computational approach described here enables a better understanding of nutrient availability and shear stresses generated in a scaffold during squeeze pressure stimulation and can be extended to other bioreactors with scaffolds and flow. Future developments and improvements could include the optimization of the pressure fields. In fact due to computational limitations, the pressure fields applied as a boundary condition did not consider their space dependency, so the pressure trends are only a function of time. Furthermore, the model matrix could be rendered more realistic by including anisotropic features or cell proliferation with consequent changes in terms of porosity, permeability, dimensions of pores, oxygen concentration and shear stress acting on cells. Finally, a time dependent fluid-structure interaction can be correlated with a corresponding time dependent oxygen consumption in the scaffold to relate scaffold degradation with cell proliferation and matrix production.

\section{Conflicts of interest}

Authors declare no conflicts of interest.

\section{Ethical approval}

Not applicable.

\section{Acknowledgments}

The work performed at University of Pisa and Politecnico di Milano was funded by the Italian Ministry of Education, University \& Research (MIUR) (Project PRIN 2010, MIND). Work developed in Pinto-do-Ó laboratory was funded by the FCT grant (PTDC/SAUORG/118,297/2010). The authors are indebted to Prof. William Clay- comb for the kind gift of the HL-1 cell-line.

\section{Supplementary materials}

Supplementary material associated with this article can be found, in the online version, at doi:10.1016/j.medengphy.2016.04. 016.

\section{References}

[1] Grayson WL, Martens TP, Eng GM, Radisic M, Vunjak-Novakovic G. Biomimetic approach to tissue engineering. Semin Cell Dev Biol 2009;20:665-73. doi:10. 1016/j.semcdb.2008.12.008.

[2] Griffith LG, Swartz MA. Capturing complex 3D tissue physiology in vitro. Nat Rev Mol Cell Biol 2006;7:211-24.

[3] Khoda AKMB, Koc B. Functionally heterogeneous porous scaffold design for tis- sue engineering. CAD Comput Aided Des 2013;45:1276-93.

[4] Zermatten E, Vetsch JR, Ruffoni D, Hofmann S, Müller R, Steinfeld A. Micro-computed tomography based computational fluid dynamics for the determina- tion of shear stresses in scaffolds within a perfusion bioreactor Ann Biomed Eng 2014;42:1085-94.

[5] Martin I, Wendt D, Heberer M. The role of bioreactors in tissue engineering. Trends Biotechnol 2004;22:80-6.

[6] Mattei G, Giusti S, Ahluwalia A. Design criteria for generating physiologically relevant in vitro models in bioreactors. Processes 2014;2:548-69. doi:10.3390/ pr2030548.

[7] Hove JR, Köster RW, Forouhar AS, Acevedo-Bolton G, Fraser SE, Gharib M. In- tracardiac fluid forces are an essential epigenetic factor for embryonic cardio- genesis. Nature 2003;421:172-7.

[8] Vunjak-Novakovic G, Radisic M, Obradovic B. Cardiac tissue engineering: effects of bioreactor flow environment on tissue constructs. J Chem Technol Biotechnol 2006;81:485-90. doi: 10.1002/jctb.1467.

[9] Santhanakrishnan A, Miller La. Fluid dynamics of heart development. Cell Biochem Biophys 2011;61:1-22. doi:10.1007/s12013-011-9158-8.

[10] Anton F, Suck K, Diederichs S, Behr L, Hitzmann B, van Griensven M, et al. Design and characterization of a rotating bed system bioreactor for tissue engineering applications. Biotechnol Prog 2008;24:140-7. doi:10.1021/bp070241b.

[11] Carrier RL, Rupnick M, Langer R, Schoen FJ, Freed LE, Vunjak-Novakovic G. Effects of oxygen on engineered cardiac muscle. Biotechnol Bioeng 2002;78:61725. doi:10.1002/bit.10245.

[12] Radisic M, Malda J, Epping E, Geng W, Langer R, Vunjak-Novakovic G. Oxygen gradients correlate with cell density and cell viability in engineered cardiac tissue. Biotechnol Bioeng 2006;93:332-43. doi:10.1002/bit.20722.

[13] Shachar M, Benishti N, Cohen S. Effects of mechanical stimulation induced by compression and medium perfusion on cardiac tissue engineering. Biotechnol Prog 2012;28:1551-9. doi:10.1002/btpr.1633.

[14] Giusti S, Pagliari F, Vozzi F, Tirella A, Mazzei D, Cabiati M, Del Ry S, Ahluwalia A. SQPR 3.0: A sensorized bioreactor for modulating cardiac phenotype. Procedia Engi 2013;59:219-25. doi:10.1016/j.proeng.2013.05.114.

[15] Cioffi M, Boschetti F, Raimondi MT, Dubini G. Modeling evaluation of the fluiddynamic microenvironment in tissue-engineered constructs: a micro-CT based model. Biotechnol Bioeng 20 06;93:50 0-10. doi: 10.1002/bit.20740.

[16] Cioffi M, Küffer J, Ströbel S, Dubini G, Martin I, WendtD. Computational evaluation of oxygen and shear stress distributions in 3D perfusion culture systems: macro-scale and micro-structured models. J Biomech 2008;41:291825. doi: $10.1016 /$ j.jbiomech.2008.07.023.

[17] Milan JL, Planell Ja, Lacroix D. Computational modelling of the mechanical environment of osteogenesis within a polylactic acid-calcium phosphate glass scaffold. Biomaterials 2009;30:4219-26. doi:10.1016/j.biomaterials.2009. 04.026 .

[18] Boschetti F, Raimondi MT, Migliavacca F, Dubini G. Prediction of the microfluid dynamic environment imposed to three-dimensional engineered cell systems in bioreactors. J Biomech 2006;39:418-25. doi: 10.1016/ j.jbiomech.2004.12.022.

[19] Lesman A, Blinder Y, Levenberg S. Modeling of flow-induced shear stress ap- plied on 3D cellular scaffolds: Implications for vascular tissue engineering. Biotechnol Bioeng 2010;105:645-54. doi: 10.1002/bit.22555.

[20] Zhao F, Vaughan TJ, Mcnamara LM. Multiscale fluid-structure interaction mod- elling to determine the mechanical stimulation of bone cells in a tissue en- gineered scaffold. Biomech Model Mechanobiol 2014;14:231-43. doi: 10.1007/s10237- 014- 0599- z.

[21] Galban CJ, Locke BR. Analysis of cell growth kinetics and substrate diffusion in a polymer scaffold. Biotechnol Bioeng 1999;65:121-32. 
[22] Galban CJ, Locke BR. Effects of spatial variation of cells and nutrient and product concentrations coupled with product inhibition on cell growth in a polymer scaffold. Biotechnol Bioeng 1999;64:633-43. doi:10.1002/(SICI) 1097-0290(19990920)64:6〈633::AID-BIT1〉 3.0.CO;2-6.

[23] Cheng G, Youssef BB, Markenscoff P, Zygourakis K. Cell population dynamics modulate the rates of tissue growth processes. Biophys J 2006;90:713-24.

[24] Obradovic B, Meldon JH, Freed LE, Vunjak-Novakovic G. Glycosaminoglycan deposition in engineered cartilage: Experiments and mathematical model. AIChE J 2000;46:1860-71. doi:10.1002/aic.690460914.

[25] Chung CA, Yang CW, Chen CW. Analysis of cell growth and diffusion in a scaffold for cartilage tissue engineering. Biotechnol Bioeng 2006;94:1138-46. doi:10.1002/bit.20944.

[26] Chung CA, Chen CW, Chen CP, Tseng CS. Enhancement of cell growth in tissue-engineering constructs under direct perfusion: Modeling and simulation. Biotechnol Bioeng 2007;97:1603-16. doi:10.1002/bit.21378.

[27] Chung CA, Chen CP, Lin TH, Tseng CS. A compact computational model for cell construct development in perfusion culture. Biotechnol Bioeng 2008;99:153541. doi:10.1002/bit.21701.

[28] De Maria C, Giusti S, Mazzei D, Crawford A, Ahluwalia A. Squeeze pressure bioreactor: a hydrodynamic bioreactor for noncontact stimulation of cartilage constructs. Tissue Eng Part C Methods 2011;17:757-64. doi:10.1089/ten.TEC. 2011.0002.

[29] Bigi A, Cojazzi G, Panzavolta S, Rubini K, Roveri N. Mechanical and thermal properties of gelatin films at different degrees of glutaraldehyde crosslinking. Biomaterials 2001;22:763-8.

[30] Spinelli A, Vinci B, Tirella A, Matteucci M, Gargani L, Ahluwalia A, et al. Re- alization of a poro-elastic ultrasound replica of pulmonary tissue. Biomater 2012;2:1-6.
[31] Claycomb WC, Lanson Na, Stallworth BS, Egeland DB, Delcarpio JB, Bahinski a, et al. HL-1 cells: a cardiac muscle cell line that contracts and retains phe- notypic characteristics of the adult cardiomyocyte. Proc Natl Acad Sci U S A 1998;95:2979-84.

[32] Hidalgo-Bastida La, Barry JJa, Everitt NM, Rose FRaJ, Buttery LD, Hall IP, et al. Cell adhesion and mechanical properties of a flexible scaffold for cardiac tissue engineering. Acta Biomater 2007;3:457-62. doi: 10.1016/ j.actbio.2006.12.006.

[33] Gonnerman Ea, Kelkhoff DO, McGregor LM, Harley BaC. The promotion of HL-1 cardiomyocyte beating using anisotropic collagen-GAG scaffolds. Biomaterials 2012;33:8812-21. doi:10.1016/j.biomaterials.2012.08.051.

[34] Porter B, Zauel R, Stockman H, Guldberg R, Fyhrie D. 3-D computational mod- eling of media flow through scaffolds in a perfusion bioreactor. J Biomech 2005;38:543-9. doi: 10.1016/j.jbiomech.2004.04.011.

[35] Raimondi MT, Boschetti F, Falcone L, Fiore GB, Remuzzi a, Marinoni E, et al. Mechanobiology of engineered cartilage cultured under a quantified fluiddynamic environment. Biomech Model Mechanobiol 2002;1:69-82. doi: 10. 1007/s10237- 002- 0007- y.

[36] Figallo E, Cannizzaro C, Gerecht S, Burdick Ja, Langer R, Elvassore N, et al. Micro-bioreactor array for controlling cellular microenvironments. Lab Chip 2007;7:710-19. doi:10.1039/b700063d.

[37] Brown MA. Pulsatile perfusion bioreactor for cardiac tissue engineering. Biotechnol Prog 2008;24:907-20. doi:10.1002/btpr.11.

[38] Brown Da, MacLellan WR, Laks H, Dunn JCY, Wu BM, Beygui RE. Analysis of oxygen transport in a diffusion-limited model of engineered heart tissue. Biotechnol Bioeng 2007;97:962-75. doi:10.1002/bit.21295. 